\title{
The influence of electronic structure modelling
} and junction structure on first-principles chiral induced spin selectivity - Supporting Information

\author{
Martin Sebastian Zöllner, ${ }^{* \dagger}$ Aida Saghatchi, ${ }^{\dagger}$ Vladimiro Mujica, ${ }^{\ddagger}$ and Carmen
} Herrmann*,†

$\dagger$ Department of Chemistry, University of Hamburg, 20146 Hamburg, Germany $\ddagger$ School of Molecular Sciences, Arizona State University, Tempe, Arizona 85287-1604, USA

E-mail: martin.zoellner@chemie.uni-hamburg.de; carmen.herrmann@chemie.uni-hamburg.de 


\section{S1 Comparison of Turbomole/X2C and ADF/ZORA for the equidistant helix}

The calculated $P_{S}$ for the equidistant perfect helical system using Turbomole/X2C and ADF/ZORA (lines 1 and 4 in Table 1 in the main manuscript) are depicted in Figure . Both methodologies give a similar shape and magnitude for $P_{S}(E)$. However, the maximum of $P_{S}$ calculated with ADF is a little bit larger than the maximum calculated with TURBOmOLE and is shifted to lower energies.

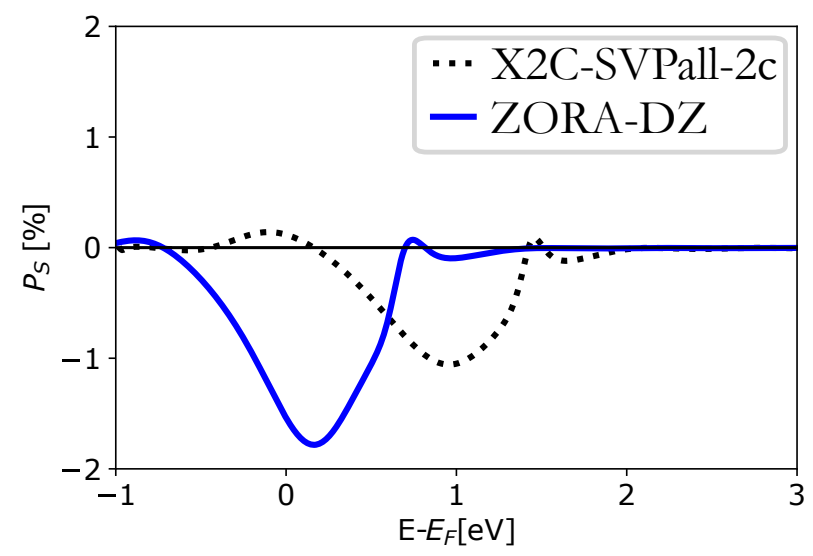

Figure S1: Comparison between $P_{S}$ calculated for the equidistant carbon helix between two 10-atom gold clusters with ADF (B3LYP/ZORA-DZ) (fourth line in Table 1 in the main manuscript) and Turbomole (B3LYP/x2c-SVPall-2c) (first line in Table 1 in the main manuscript). $P_{S}$ is shifted and larger for ADF, but both calculations show a similar magnitude for $P_{S}$. 


\section{S2 Complete Hamiltonian matrix for different admix- tures of Hartree-Fock exchange}

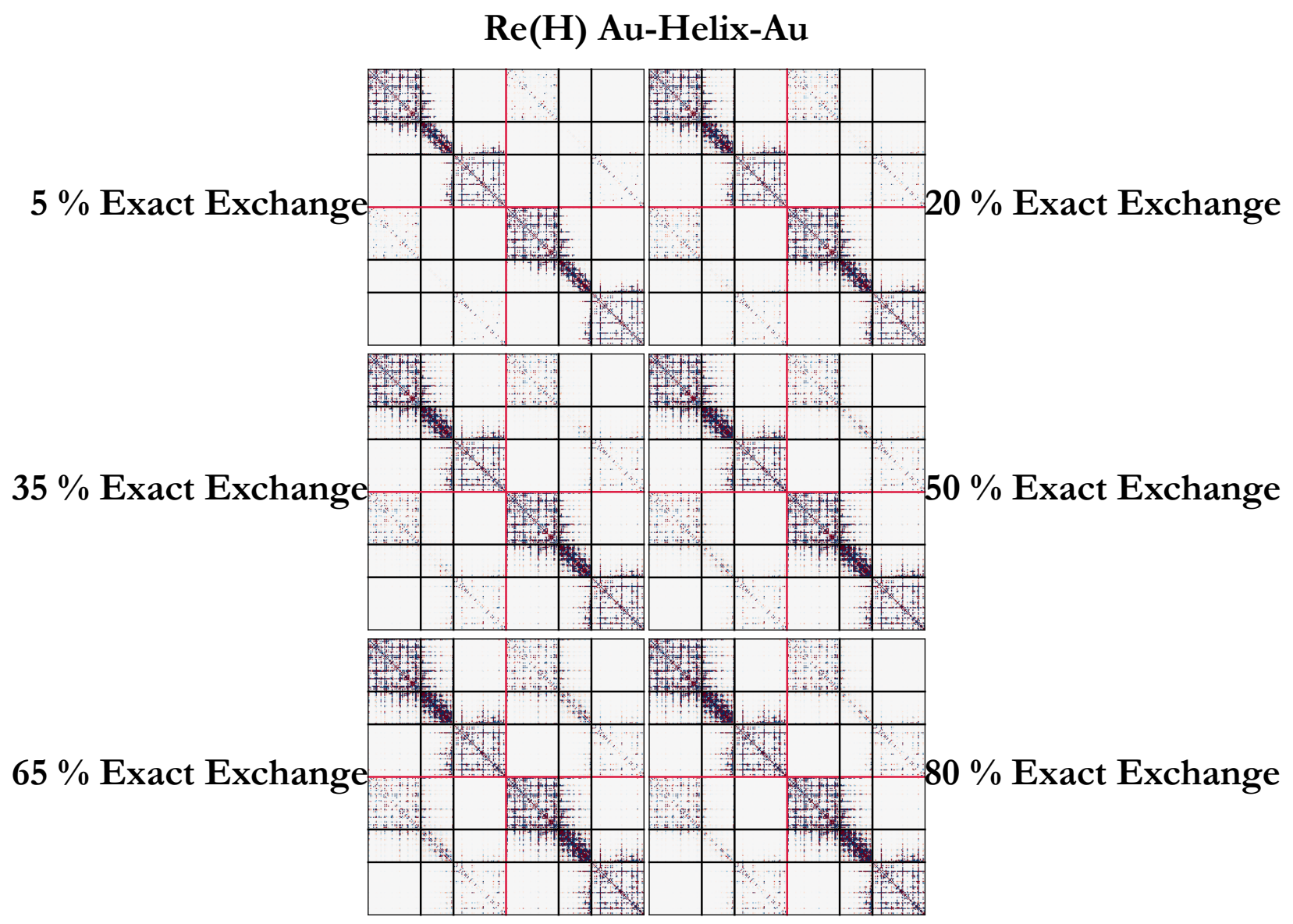

Figure S2: $\operatorname{Re}(\mathbf{H})$ for the equidistant perfect carbon helix between two 10-atom gold clusters, calculated for several admixtures of exact exchange (from top left to bottom right: $5 \%, 20 \%, 35 \%$, $50 \%, 65 \%, 80 \%)$. The red lines separate the four different spin blocks, the black lines separate the different regions of the junction (left, central, right). Up to $50 \%$, an increase of the coupling matrices can be observed. However, no significant decrease can be observed for admixtures higher than $50 \%$. Thus, these matrices cannot explain the decrease of the inherited SOC when the Hartree-Fock exchange is increased to $60 \%$ and $80 \%$. 


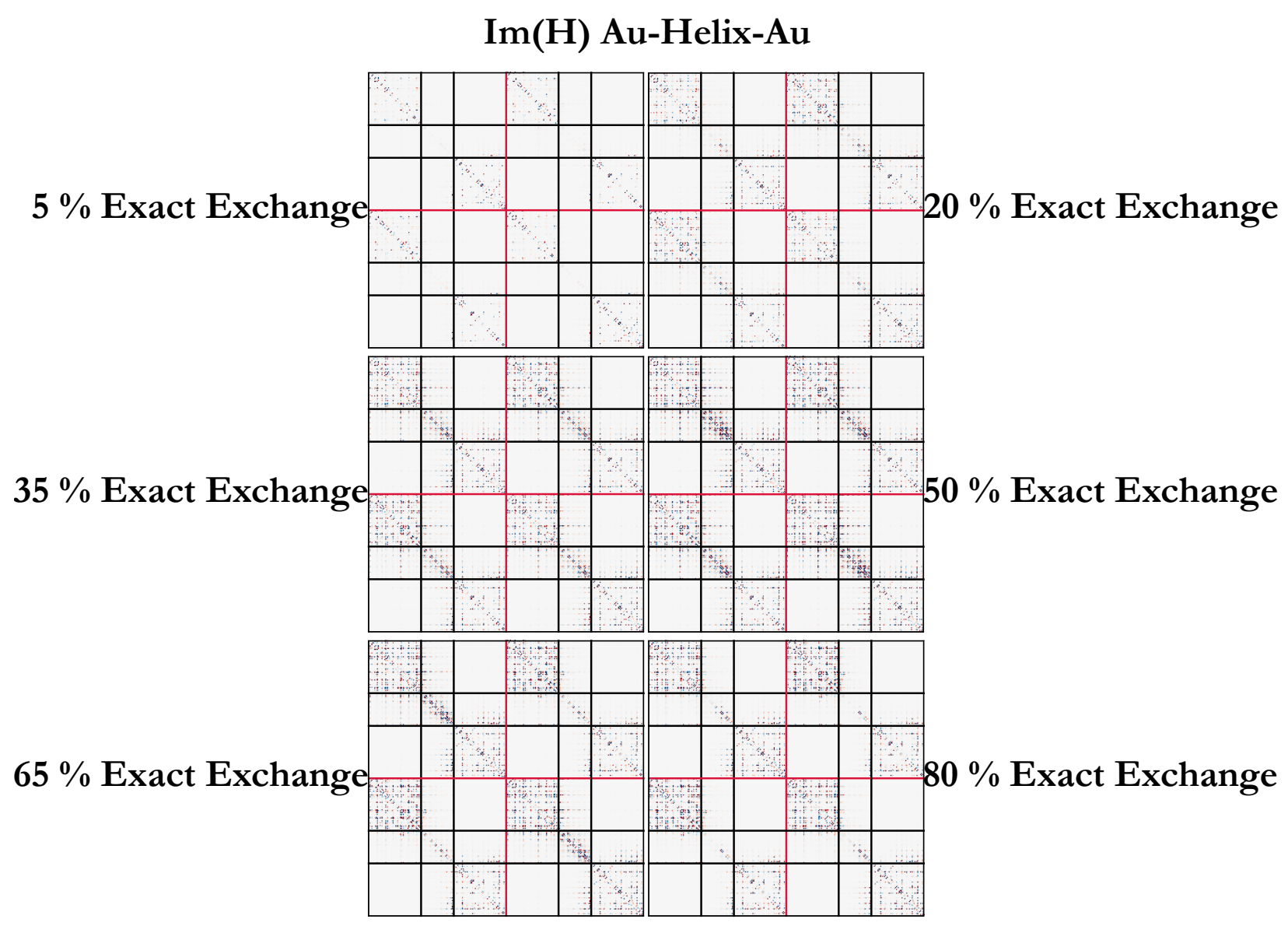

Figure S3: $\operatorname{Im}(\mathbf{H})$ for the equidistant perfect carbon helix between two 10-atom gold clusters, calculated for several admixtures of exact exchange (from top left to bottom right: $5 \%, 20 \%, 35 \%$, $50 \%, 65 \%, 80 \%)$. The red lines separate the four different spin blocks, the black lines separate the different regions of the junction (left, central, right).

\section{S3 Energy levels of the central and electrode subsys- tems}

To gain further insight into the dependence of $P_{S}$ on the amount of exact exchange, we calculated the effective single-particle energy levels (molecular orbitals) of each subsystem of the junction (left or right electrode, molecule $=$ central region). This is done by solving the secular equation determining the levels / orbitals of each subsystem independently.

The amount of exact exchange shifts the energy levels of the central region and both electrode regions. However, there is no clear correlation between the level alignment of the electrode 
/ central region and $P_{S}$.

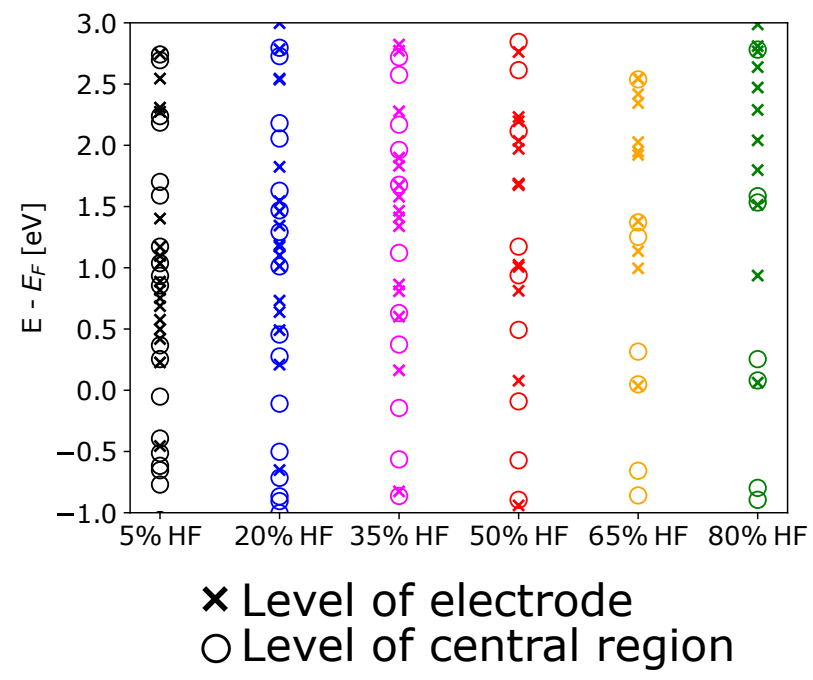

Figure S4: Energy levels of the central region (o) and left and right electrodes (x), calculated for each subsystem separately. The levels shift when the amount of Hartree-Fock exchange is increased, however, no clear hint of, on the one hand, an increase of level alignment up to $50 \%$ and, on the other hand, a decrease for amounts of exact exchange larger than $50 \%$ can be observed.

\section{S4 Helix with alternating bond pattern and different hydrogen placement}

To investigate the effect of the hydrogen orientation on $P_{S}$ for the alternating helix, we calculated $P_{S}$ for an alternating helix with an adapted hydrogen orientation.

The hydrogen atoms were adapted in the following way: for the hydrogen atoms at the starting point of the helix (top carbon atom in Figure S6, position $\mathrm{x}=0, \mathrm{y}=0, \mathrm{z}=0$ for both bond patterns), we used the same hydrogen coordinates for the equidistant and alternating helix. This results in the same distances of the terminal carbon with the hydrogen atoms, the same angle between the hydrogen atoms and the terminal carbon atom, and the same height difference. We then added the hydrogen atoms at the end point of the helix, and optimized their position by minimizing the difference of certain internal coordinates between the hydrogen atoms and the three closest carbon atoms, using the contact at the starting 
point of the helix as a reference. The used internal coordinates were the distances between the hydrogen atoms, the distances of the hydrogen atoms and the three closest carbon atoms, and the dihedral between the hydrogen atoms and the second and third nearest carbon atoms. The resulting structure was used to investigate the effect of the contact region for the alternating helix. The xyz coordinates are also provided within a zip file.

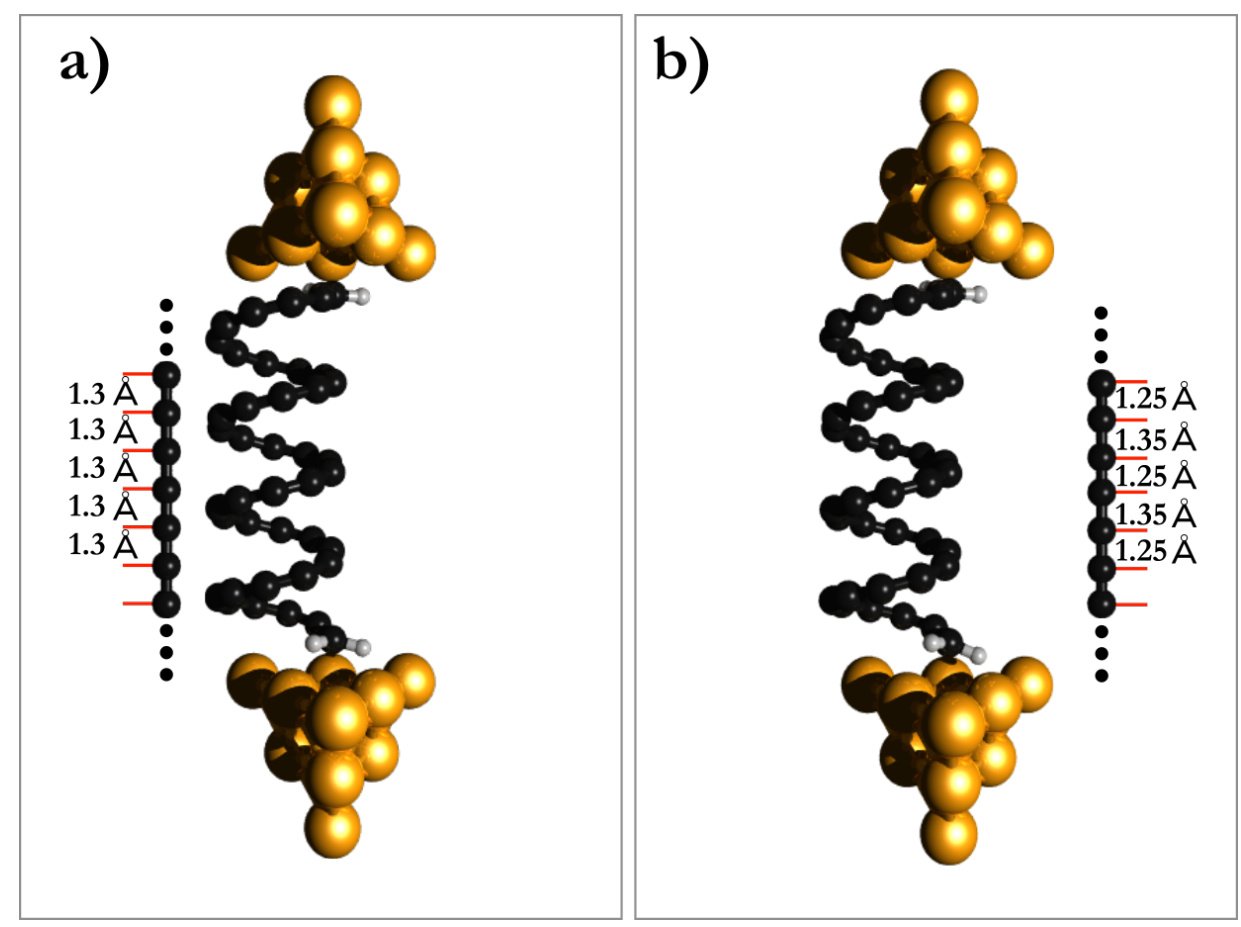

Figure S5: Structures of the gold-molecule-gold junction of the carbon helix with a) equal and b) alternating bond distances with an alternative hydrogen placement. 

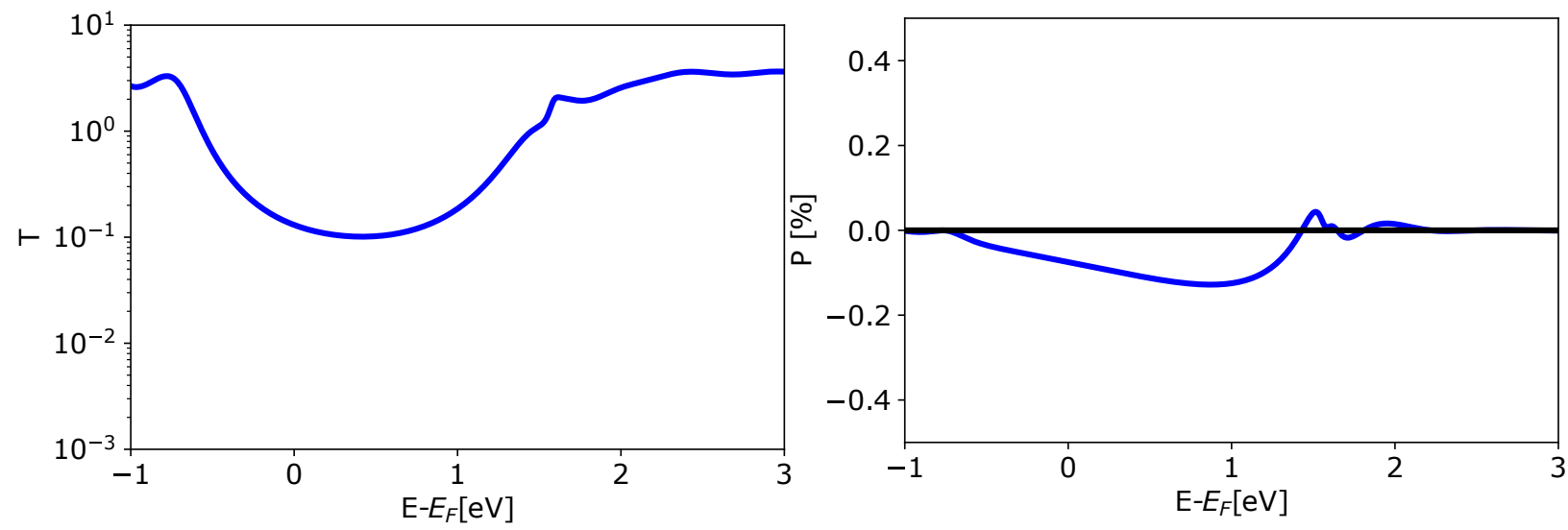

Figure S6: Transmission function and $P_{S}$ calculated for the perfect helix with alternating bond pattern. In contrast to the main text, the hydrogen atoms are oriented differently (see text), leading to a different contact region. Again, $P_{S}$ for the alternating helix is much smaller than $P_{S}$ for the equidistant helix. However, the contact region, i.e., the hydrogen orientation appears to influence $P_{S}$ for the alternating helix as well.

\section{S5 Effect of an electric field}

In conductance experiments, measurements of the CISS effect are done by applying a finitesize voltage. Thus, it cannot be excluded that the equilibrium zero-bias approach used in this work misses crucial aspects in the underlying mechanism. Although it is not possible within our methodology to simulate a true non-equilibrium situation by applying a voltage, we can approximate the electric potential within the junction with a linear electric field. The results indicate (see Figure S7) that the linear field does not influence $P_{S}$ strongly. This may change when an explicit non-equilibrium description of the electronic structure is included. 


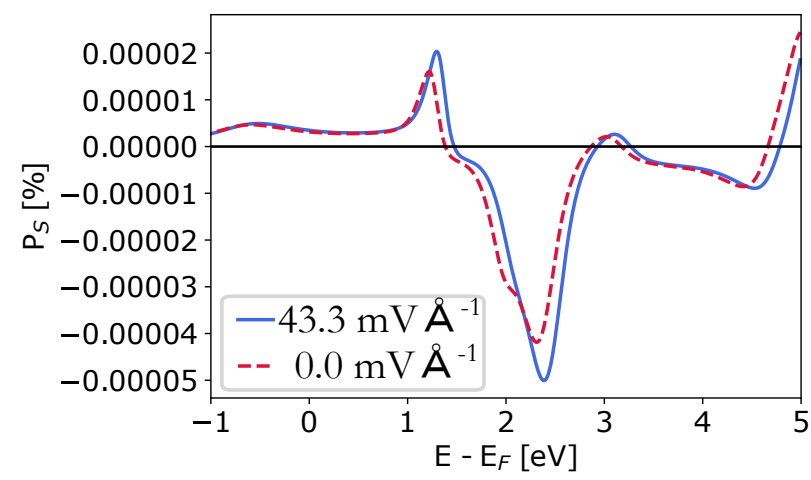

Figure S7: Calculated $P_{S}$ (B3LYP/ZORA-DZ) for the gold-helicene-gold junction with and without an applied electric field $\left(43.3 \mathrm{mV} \AA^{-1}\right)$. The effect of the electric field is very small.

\section{S6 Varying the copper-copper distance}

In the main manuscript, we had set the copper-copper distance to the same $2.88 \AA$ as for gold, for simplicity and for a direct comparison. The measured copper-copper distance in the metal is $2.55 \AA .1$ To evaluate the effect of this distance, we have evaluated polarization for the 40-atom evenly spaced carbon helix attached to 10-atom copper clusters with $2.55 \AA$ copper-copper distances, employing Turbomole with an x2c-SVPall-2c basis set and relativistic effects taken into account by the X2C method (see first line of Table 1 in the main manuscript). The shorter distances do not change the polarization substantially, and importantly they do not change our conclusions. 


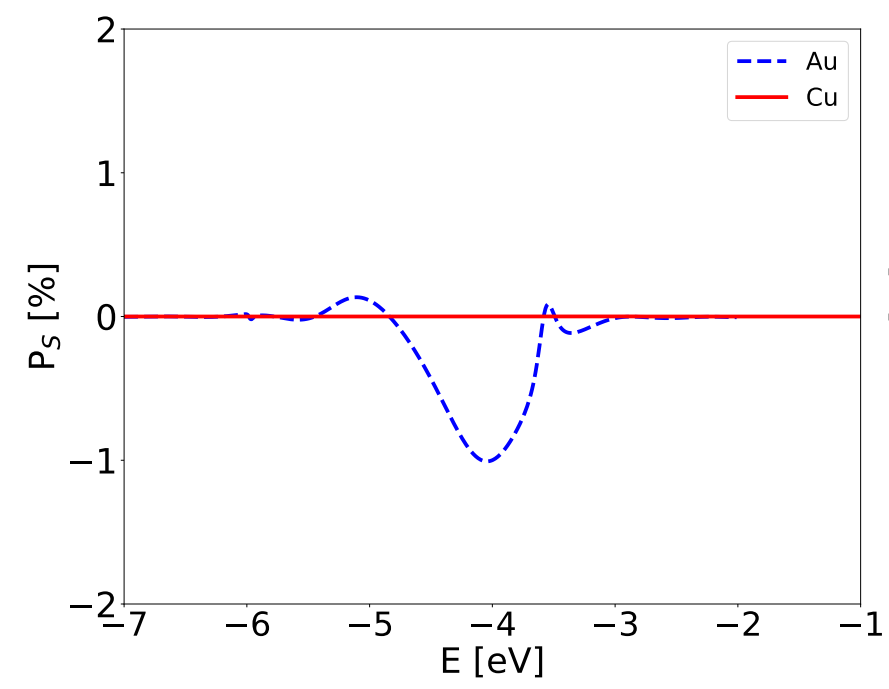

(a)

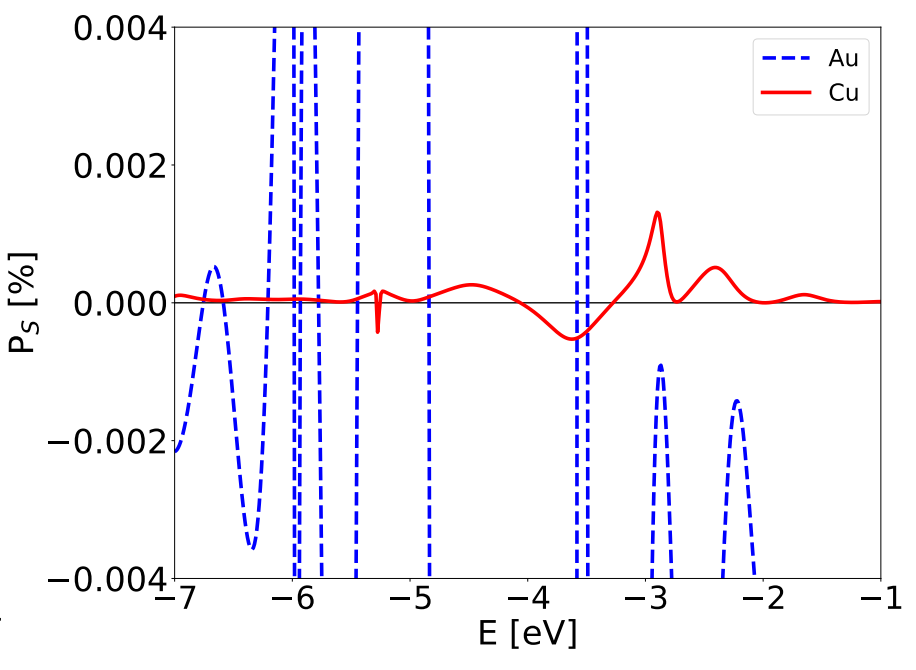

(b)

Figure S8: Spin polarization for different cluster metals ( $\mathrm{Au}$ and $\mathrm{Cu}$ in one plot) and 40 carbon (a) full range of y axis. (b) limited range of y axis. In contrast to the main manuscript, these results are not shifted with respect to the Fermi energy, which we had set to $-5 \mathrm{eV}$.

For comparison, we have also looked at a shorter helix with 20 carbon atoms evenly spaced by 1.3 (Figure S12), along a perfect helical path with a pitch of 3 and a radius of 2 , constructed in agreement with the 20-carbon-atom junction in Ref., 2 attached to the same copper clusters with $2.55 \AA$ copper-copper distances. The computational settings for the short helix were again as in the first line of Table 1 in the main manuscript, except that here TuRBOmole version 7.4.1 rather than 7.1 was used. We do not expect the change in version to affect our results. In contrast to the gold electrodes, the change in length affects mostly the shape of the polarization, but there is no clear decrease in magnitude. This could suggest that the strong SOC transfer from gold, though length-dependent, dominates over more subtle effects which become visible here. 


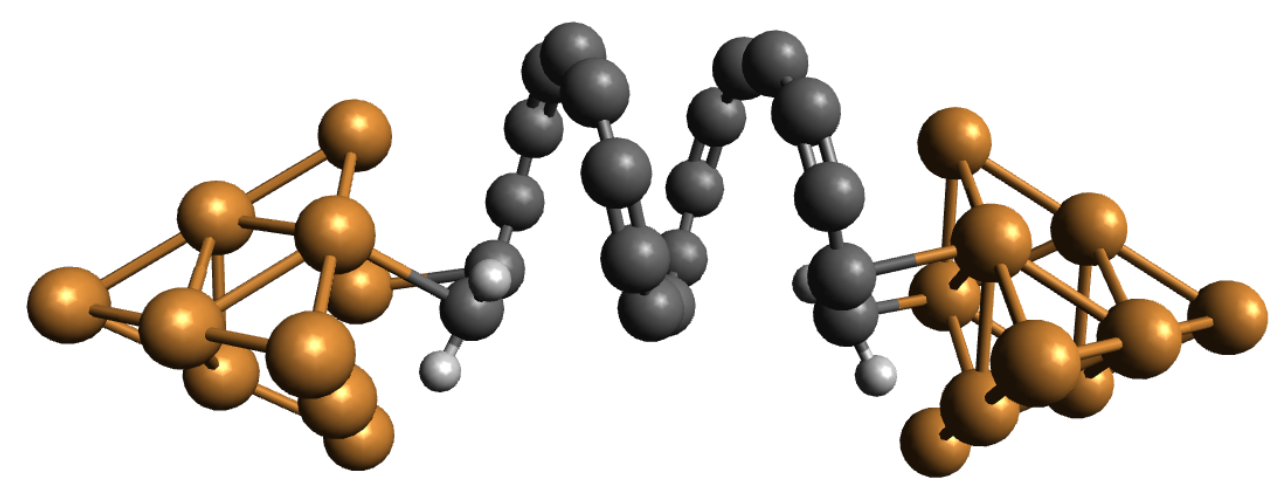

Figure S9: Structure of the coppermoleculecopper junction of the 20-atom carbon helix, for electrode clusters with $2.55 \AA \mathrm{Cu}-\mathrm{Cu}$ distances.

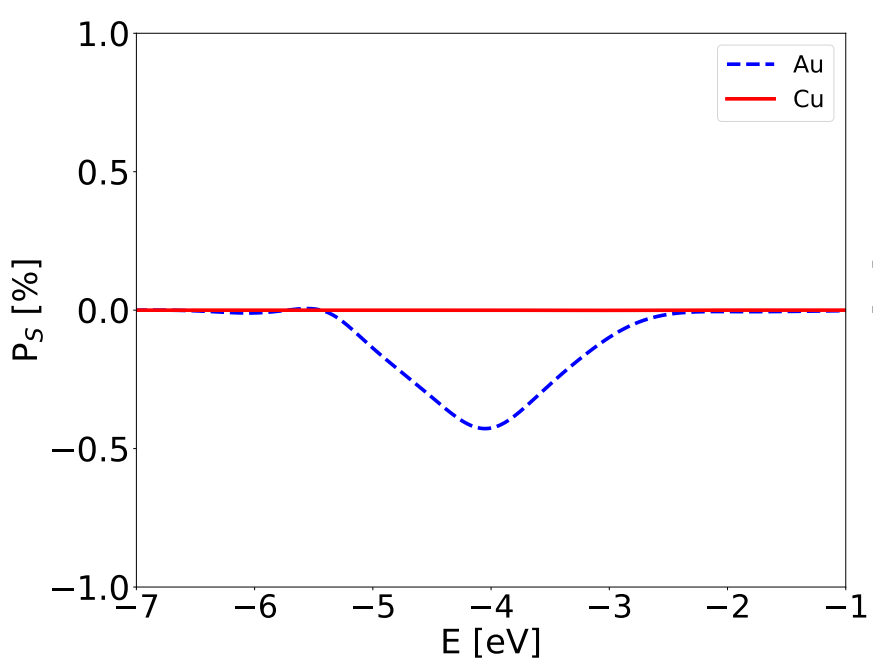

(a)

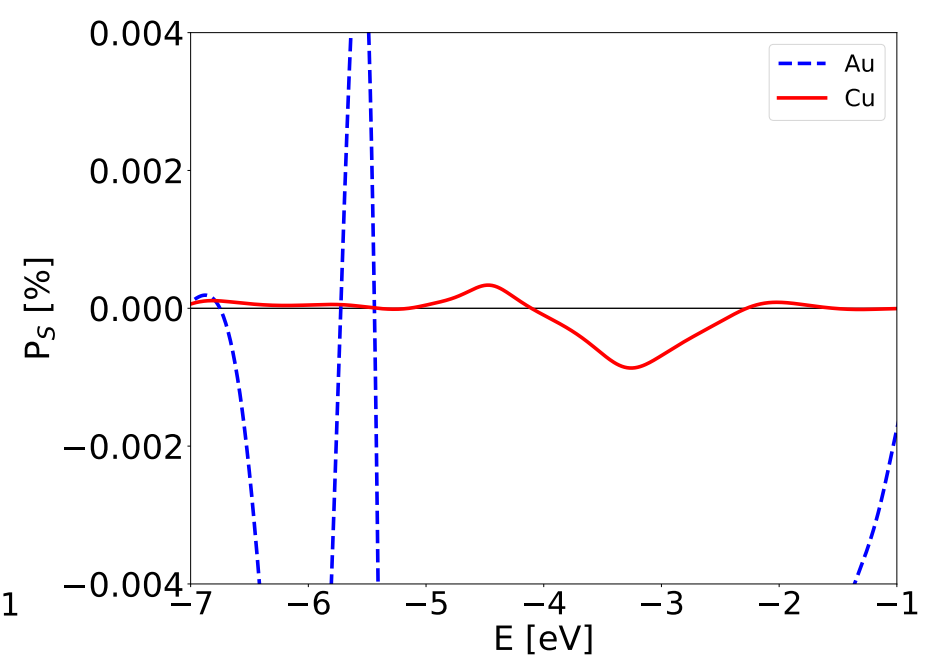

(b)

Figure S10: Polarization for a 20-atom helix for $\mathrm{Au}$ and $\mathrm{Cu}$ electrodes, employing 6-3-1 10-atom clusters (a) Full plot, (b) zoom-in.

\section{S7 Varying the helix-electrode distance}

To check for the importance of electrode-molecule interactions, we carried out calculations for 20-atom helices with the carbon-gold distances increased from 2.48 to 2.58, 2.68, and

$4.48 \AA$, respectively (structures and settings see Section S6). The polarization decreases 
substantially when increasing the distance between helix and electrode. In the imaginary parts of the effective single-particle Hamiltonian matrices, the changes resulting from the distance variations by $0.1 \AA$ are small, at least compared to the absolute values of the matrix elements. If the distance is increased substantially, to $4.48 \AA$, a clear decrease is visible, however.

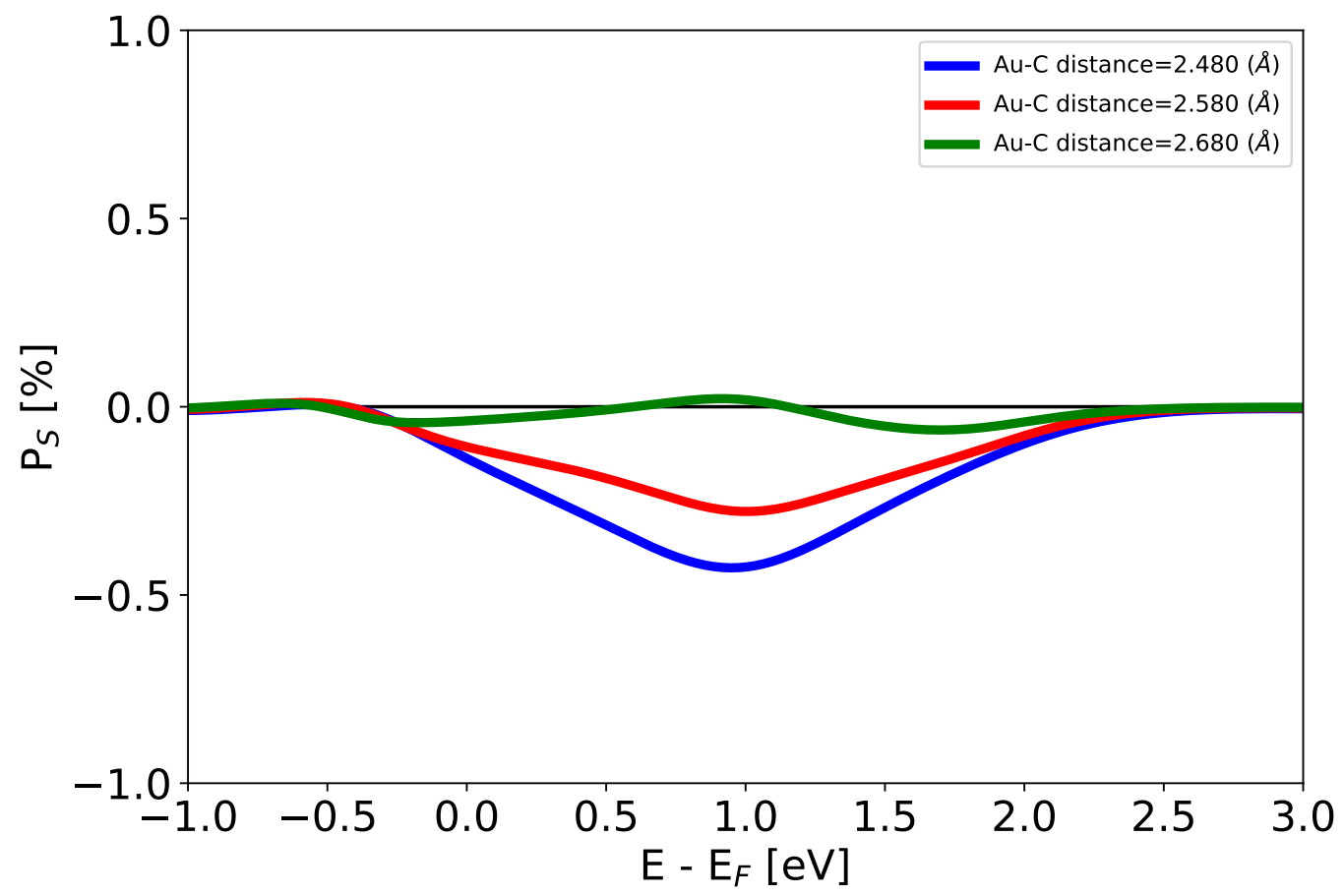

Figure S11: Polarization as a function of carbon-gold distance for a 20-atom carbon helix. 


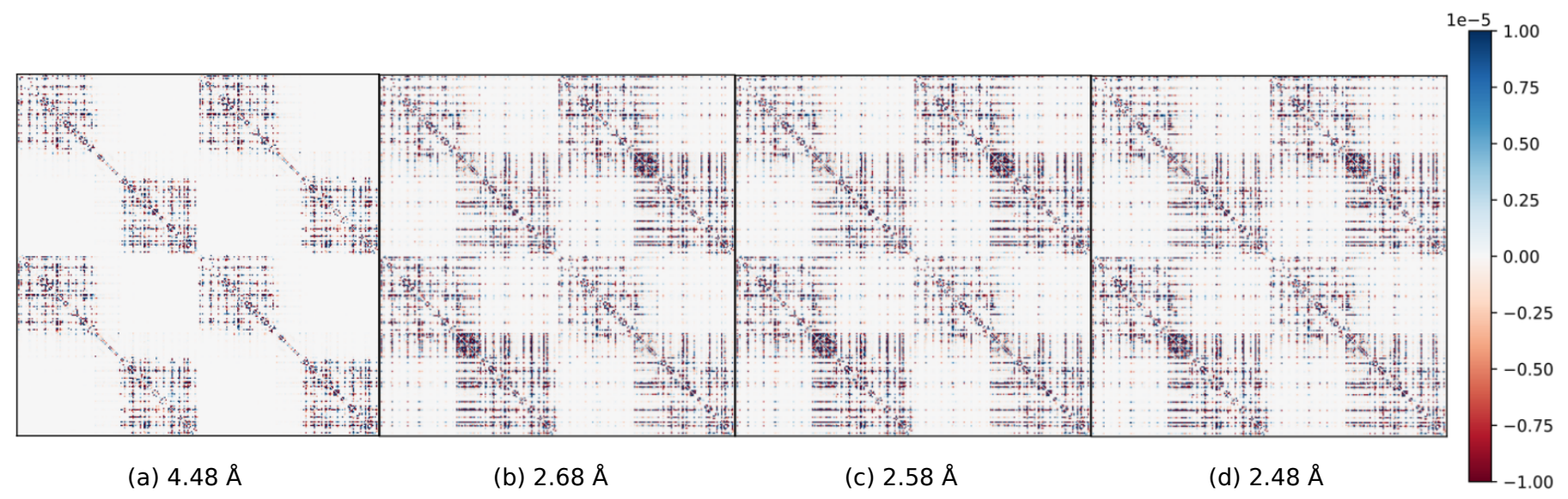

Figure S12: $\operatorname{Im}(\mathrm{H})$ for gold-carbon-gold junction. From right to left distances of gold-carbon alter from 2.48 to $2.58,2.68$ and 4.48 .

\section{S8 Transmission functions}

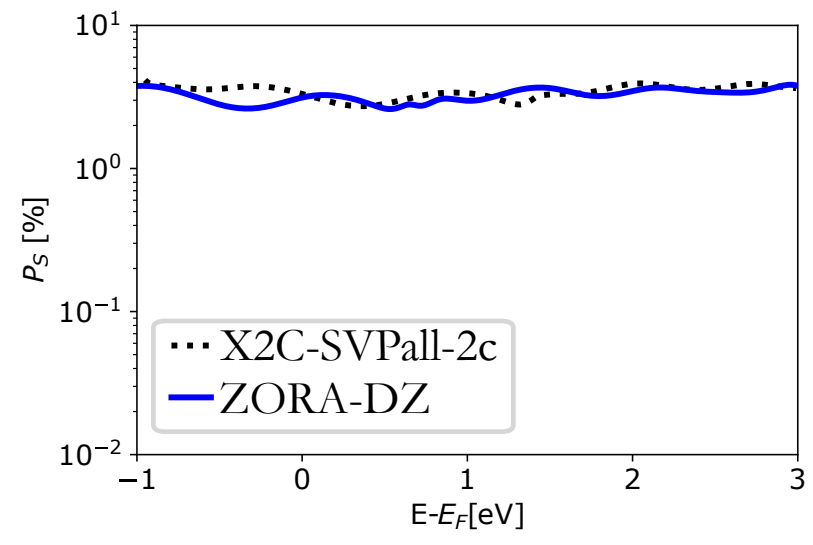

Figure S13: Transmission function of the equidistant carbon helix between two 10-atom gold clusters calculated with ADF (B3LYP/ZORA-DZ) and TURBOmOLE (B3LYP/x2c-SVPall2c). Both transmission functions are quite similar and only slightly shifted. 


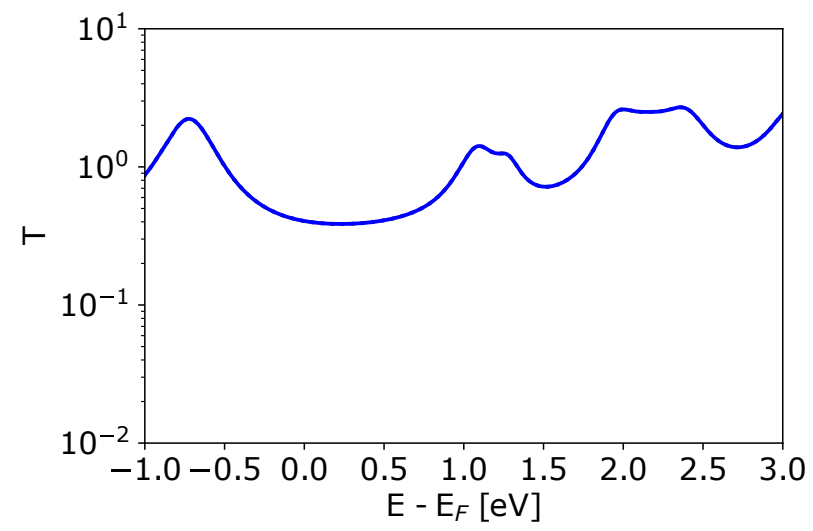

Figure S14: Calculated transmission function (B3LYP/ZORA-DZ) of the gold-helicene-gold junction.

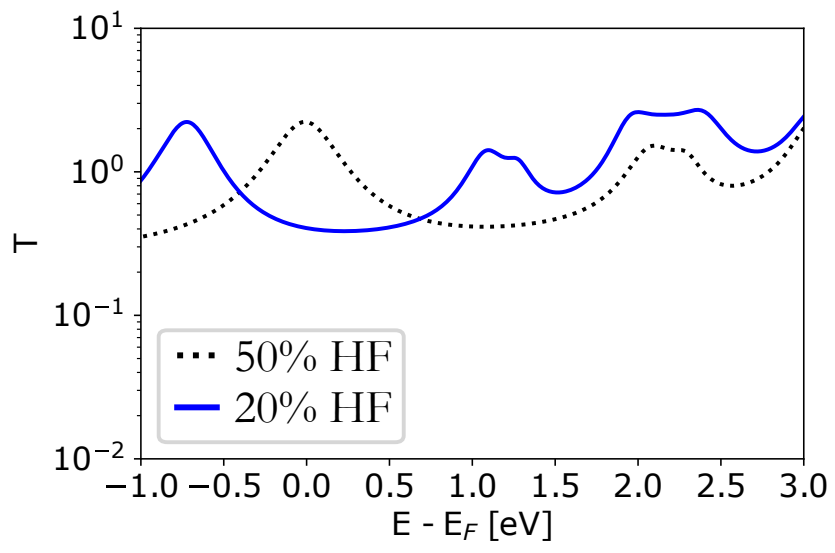

Figure S15: Transmission function calculated for the gold-helicene-gold junctions (B3LYP/ZORA-DZ) using two different amounts of exact exchange (20 \% and $50 \%)$. Increasing the amount of exact exchange shifts the transmission function to higher energies.

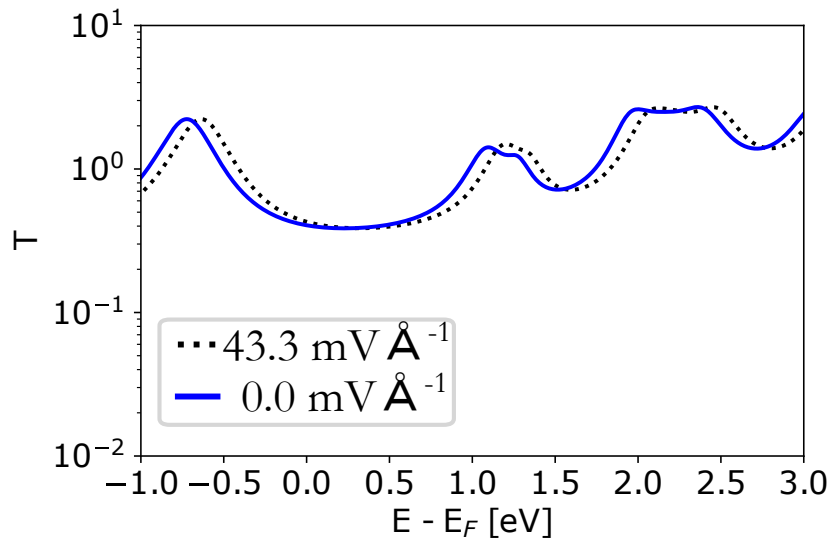

Figure S16: Calculated transmission function (B3LYP/ZORA-DZ) for the gold-helicenegold junction, with and without electric field. The electric field slightly shifts the transmission function. 


\section{S9 Preferred spin state of the used gold clusters}

Table S1: Difference of energy for the triplet and singlet state for several used gold clusters. We used the energy difference of the total energies for TURBOMOLE and the energy differences of the bond energies for ADF. A positive energy difference indicates the triplet state to be favored.

\begin{tabular}{r|c}
\hline \hline Cluster & $\Delta\left(E_{S}-E_{T}\right)[e V]$ \\
\hline $6-3-1(\mathrm{e} 2-S V P a l l-2 \mathrm{c})$ & 0.57 \\
$6-3-1$ (dhf-SVP) & -0.01 \\
$16-0-0$ (dhf-SVP) & 0.24 \\
$16-16-0$ (dhf-SVP) & 0.24 \\
$16-16-16$ (dhf-SVP) & 0.20 \\
$10-6-3-1$ (ZORA-DZ) & -1.81 \\
\hline \hline
\end{tabular}

\section{References}

(1) Simon, N. J.; Drexler, E. S.; Reed, R. P. Properties of copper and copper alloys at cryogenic temperatures. National Inst. of Standards and Technology, Monograph 177. 1992.

(2) Zöllner, M. S.; Varela, S.; Medina, E.; Mujica, V.; Herrmann, C. Insight into the Origin of Chiral-Induced Spin Selectivity from a Symmetry Analysis of Electronic Transmission. J. Chem. Theory Comput. 2020, 16, 2914-2929, ChemRxiv, DOI: 10.26434/chemrxiv.8325248.v2. 